\section{The visibility of non-communicable diseases in Northern Uganda}

Susan Reynolds Whyte ${ }^{1}$, Sung-Joon Park², George Odong ${ }^{3}$, Moris Ojara ${ }^{3}$, Alice Lamwaka ${ }^{4}$

1. Department of Anthropology, University of Copenhagen, Denmark

2. Institute of Anthropology, University of Leipzig, Germany

3. Faculty of Medicine, Gulu University, Uganda

4. Department of Pharmacy, Faculty of Medicine, Gulu University, Uganda

\section{Abstract}

Background: WHO and Uganda's Ministry of Health emphasize the need to address the growing burden of non-communicable diseases (NCDs). Treatment for these conditions is urgent in northern Uganda where war has negatively affected both health and the public health care system.

Objectives: We aimed to explore the recognized presence of selected chronic conditions in the out-patient population and to relate this 'visibility' to the ability of health units to diagnose and treat them.

Methods: At six health facilities we reviewed patient registers for one month to determine the frequency of hypertension, diabetes, depression, and post traumatic stress disorder (PTSD). We checked the availability of diagnostic instruments and medicines, and interviewed health workers.

Results: The four conditions were rarely diagnosed in the outpatient population. Hypertension was the most common, but still constituted under $1 \%$ of diagnoses. Patterns of dia outpatient population. Hypertension was the most connon, bu still constituted under $1 \%$ of diagnoses. Patterns of diagnosis were uneven, with higher frequency of particular diagnose at some health facilities. Diagnostic equipment was not sufficient and screening was irregular. Medicine was mostly available
although stockouts of some relevant drugs were reported. Conclusions: The four conditions are relatively invisible in the outpatient population. Greater visibility would be facilitated by regular clinic days for hypertension and diabetes, availability and regular use of diagnostic instruments, and a more reliable supply of the relevant medicines.

Keywords: non-communicable diseases, Northern Uganda

DOI: http://dx.doi.org/10.4314/ahs.v15i1.11

\section{Introduction}

Non-communicable diseases represent an increasing burden, also in countries that have high rates of infectious diseases. ${ }^{1,2}$ The WHO global strategy for NCDs and an implementation plan were launched in the first decade of the new millennium with a particular focus on cardiovascular disease, diabetes, chronic lung diseases, and cancer. ${ }^{3}$ In Uganda, NCDs were made a national priority in 2006 when they were incorporated in

\section{Corresponding author:}

Susan Reynolds Whyte

Department of Anthropology

University of Copenhagen

Oester Farimagsgade 5

DK-1353 Copenhagen, Denmark

Tel: +4535323477

Email: susan.reynolds.whyte@anthro.ku.dk

es and mental health 2008-2013 calls for improvement in management of NCDs and integration of mental health into primary health care. ${ }^{8}$
As northern Uganda recovers from two decades of war, its public health care system is being rebuilt with important contributions from NGOs, bilateral and multilateral donors. However, this support continues to be earmarked for certain health conditions, especially HIV. Given the high priority policymakers attribute to NCDs and the challenges of impaired health and weakened health care systems in northern Uganda, as well as the need for research on NCDs in primary health care, ${ }^{9}$ we ask how well public health facilities there are addressing chronic conditions. To what extent are they visible in the outpatient population? Since their (in)visibility is in part a function of the capacity of facilities to identify them and provide medication, it is necessary to review the availability of diagnostic instruments and assess the diagnostic routines and appropriate medication.

\section{Methods}

Between November 2011 and February 2012, a team based at Gulu University made an explorative study of the frequency and treatment of chronic conditions in six health facilities purposively chosen from different districts and with different levels of service. There were three Health Centres level 3 (HC3) and two Health Centres level 4 (HC4), as well as one private not-for-profit (PNFP) rural hospital functioning as a district hospital. They were located in Gulu (1), Amuru (1), Nwoya (1), and Agago (2) Districts, and a division of Gulu Municipality (1).

We focused on out-patient diagnosis and medication for four health conditions: hypertension, diabetes, depression, and post traumatic stress disorder (PTSD). The first two were selected because they figure in the new global policy for NCDs and because they are thought to be highly prevalent or increasing in Uganda. Depression and PTSD were chosen because many health workers, policymakers and researchers see them as high priority in northern Uganda after the years of war and violence.

The study adopted mixed methods - mostly qualitative with some quantitative elements. On the quantitative side, we obtained Health Management Information System (HMIS) figures on cases of the four conditions reported in two districts over five years. At each health facility, we examined the patient register books and recorded numbers diagnosed with the four conditions and medicines prescribed for them during the month of October 2011 in the Out-Patient Department (OPD).
We noted prescription of amitriptyline for diagnoses ther than depression and PTSD. Against a checklist, we recorded the availability of functioning blood pressure (BP) machines, glucometers and strips, and diagnostic guidelines for depression and PTSD, such as Uganda National Treatment Guidelines, Beck's Depression Inventory, and the Harvard Trauma Questionnaire. We inspected the store to see which medicines were in stock for these four conditions. For both instruments and medicines we considered what should have been available at different levels of health facility. (Health Centres 3 receive their medicine supplies on the 'push' system; they are supplied with kits containing fixed types and amounts of drugs. Health Centres 4 and hospitals are on the 'pull' system; they can order the drugs they need from National Medical Stores [NMS], if they are available.)

The qualitative data came from open-ended interviews We discussed the treatment of NCDs with two distric health officers. At each of the six health facilities, we interviewed in-charges and health facility staff on topics including the following: levels of staffing and formal training (e.g. psychiatric nurses); patterns of use of the above diagnostic instruments; designated clinics for HIV, mental health, and NCDs; experiences and challenges in treating the four conditions. Given the importance of external funding for health care in Uganda, we asked about sources of external support for the facility and the conditions targeted. We interviewed 9 patients on a convenience basis, at least one from each facility.

We also explored two areas of activity beyond the health facilities, but relevant to the recognition and treatment of NCDs. Since patients are told to buy medicines when the public facility is stocked out, we made explorative visits to 8 drugshops and small private clinics within $500 \mathrm{~m}$ of two of the health facilities and to four pharmacies and private clinics in Gulu town. We checked whether they sold medicines for these diagnoses and sold or used the relevant diagnostic instruments. In view of the intense attention to mental trauma due to the war and the effect this might have on recognition in health facilities, we made a rapid mapping of NGOs and donors active in relation to trauma conditions using a structured questionnaire

The interview and observation material was transcribed from field notebooks and coded manually. We were given permission to examine records at each facility by the (acting) in-charge. Informed consent was obtained from every individual interviewed.

African Health Sciences Vol 15 Issue 1, March 2015 
As in the rest of the coutry, there are no popula- also sow. By the com A in the five years, hypertension Ureve figures for NCDs in northern numbers were similar to those for HIV. Nevertheless, ganda. However HMIS reports for a five-year period hypertension constituted only $0.8 \%$ of all diagnoses for in Gulu district (Table 1) reveal a substantial increase outpatients age 5+ in 2010/11 even though it was the most frequent of the categories examined.

\section{Table 1. Selected chronic disease diagnoses in Gulu District for outpatient} age 5+ Source: District HMIS data

\begin{tabular}{|l|l|l|l|l|l|}
\multicolumn{1}{c}{$2006 / 7$} & \multicolumn{1}{c}{$2007 / 8$} & \multicolumn{1}{c}{$2008 / 9$} & \multicolumn{1}{c}{$2009 / 10$} & \multicolumn{1}{l}{$2010 / 11^{* *}$} \\
\hline HT $^{*}$ & $1099 / 2555$ & $1056 / 2299$ & $1326 / 3036$ & $1080 / 3465$ & $1117 / 3314$ \\
\hline DM* & $193 / 258$ & $555 / 1106$ & $475 / 682$ & $183 / 264$ & $412 / 758$ \\
\hline Depression & $148 / 266$ & $134 / 348$ & $326 / 843$ & $395 / 1181$ & $432 / 1672$ \\
\hline HIV & $1428 / 2497$ & $2197 / 3650$ & $3075 / 4065$ & $1984 / 2673$ & $1920 / 3155$ \\
\hline
\end{tabular}

\section{Male/Female}

${ }^{*} \mathrm{HT}=$ hypertension, $\mathrm{DM}=$ diabetes mellitus

$* *$ total diagnoses for all outpatients age $5+$ for $2010 / 11=535,897 ; \mathrm{HT}=0.8 \%$ of all diagnoses

As shown in Table 2, the pattern for Amuru does not pertension and depression were reported in 2007/8 and have the same clear increase; the highest figures for hy- for diabetes in 2008/9.

Table 2. Selected chronic disease diagnoses in Amuru District for outpatients age 5+ Source: District HMIS data

\begin{tabular}{|l|l|l|l|l|l|}
\multicolumn{1}{l}{$2006 / 7$} & \multicolumn{1}{c}{$2007 / 8$} & \multicolumn{1}{c}{$2008 / 9$} & \multicolumn{1}{l}{$2009 / 10$} & $2010 / 11$ \\
\hline HT & No Data* & $186 / 576$ & $149 / 296$ & $113 / 501$ & $87 / 471$ \\
\hline DM & No Data & $9 / 10$ & $57 / 85$ & $28 / 53$ & $31 / 69$ \\
\hline Depression & No Data & $66 / 83$ & $17 / 46$ & $15 / 69$ & $9 / 37$ \\
\hline HIV & No Data & $434 / 737$ & $304 / 429$ & $545 / 924$ & $431 / 780$ \\
\hline
\end{tabular}

\section{Male/Female}

Amuru District was not established until 2006 and lacked HMIS data for the first year.

In both district reports, hypertension and depression At the individual health facilities, patterns of diagnosi were at least twice as frequent for women, while diabe- varied. Table 3 shows diagnoses for outpatients age 5 tes was more common for men.

Table 3. Selected chronic disease diagnoses for outpatients age 5+ October 2011

\begin{tabular}{l|l|l|l|l|l|}
\multicolumn{1}{c}{ Health unit } & \multicolumn{1}{c}{ HT } & \multicolumn{1}{c}{ DM } & \multicolumn{1}{c}{ Depression } & \multicolumn{1}{c}{ PTSD } & Total pts* \\
\hline HC3 a & 4 & 0 & 6 & 0 & 720 \\
\hline HC3 b & 12 & 0 & 0 & 0 & 4079 \\
\hline HC3 c & 9 & 0 & 2 & 4 & 1700 \\
\hline HC4 a & 12 & 3 & 29 & 1 & 1461 \\
\hline HC4 b & 1 & 3 & 0 & 0 & 2425 \\
\hline PNFP hosp & 24 & 21 & 3 & 0 & 940 \\
\hline
\end{tabular}

* Total recorded outpatients age $5+$ for October 2011

Hypertension was the most common of the four con- For diagnosing diabetes a glucometer is preferred to ditions, but still constituted under $1 \%$ of all out-patient diagnoses except at the private not-for-profit hospital where it comprised $2.5 \%$ of total OPD diagnoses for age $5+$. Diabetes was not diagnosed at any HC3, and only rarely at the two HC4, while one HC4 had almost twice as many depression diagnoses as all the other facilities combined. PTSD was diagnosed only 5 times in all, with 4 of the cases identified at one $\mathrm{HC} 3$.

Diagnostic instruments

All facilities visited had blood pressure (BP) meters, but there were differences in how regularly they were available in OPD and how systematically BP was measured. In-charges emphasized that mothers enrolling for antenatal clinic (ANC) should have their BP checked at the first visit. One $\mathrm{HC} 3$ had only one BP meter, provided to the ANC by Northern Uganda Malaria, AIDS and Tuberculosis Program in connection with the prevention of mother to child transmission activities. This machine usually stayed in OPD and was borrowed by ANC when needed. Another HC4 had two BP meters that were kept by clinical officers. When they were not on duty, no BP meter was available. Health workers stated that they only checked outpatients with signs and symptoms suggestive of hypertension such as severe headache, palpitation, and burning sensations in the lower limbs. Several thought that BP should be measured for more patients, but that took extra time, and the BP machine was not always present in OPD. When the meters broke down, it was difficult to get them repaired since the donor program supporting equipment maintenance had ended. All facilities visited had broken BP machines in their stores.

Medicine

Table 4 shows the drugs in stock at each unit for the four conditions. Depression and PTSD are treated with the same medications, Amitriptyline and Imipramine, so they are amalgamated in the table. 


\section{Table 4 .Medications in stock for selected chronic conditions January 2012}

\begin{tabular}{|c|c|c|c|}
\hline $\mathrm{HC} 3 \mathrm{a}$ & Propranolol & none & $\begin{array}{l}\text { Amitriptyline } \\
\text { Imipramine }\end{array}$ \\
\hline $\mathrm{HC} 3 \mathrm{~b}$ & Propranolol & Glibenclamide* & none \\
\hline $\mathrm{HC} 3 \mathrm{c}$ & Propranolol & none & none \\
\hline $\mathrm{HC} 4 \mathrm{a}$ & $\begin{array}{l}\text { Nifedipine } \\
\text { Propranolol } \\
\text { Atenolol } \\
\text { Furosemide } \\
\text { Methyldopa }\end{array}$ & none & $\begin{array}{l}\text { Amitriptyline } \\
\text { Imipramine }\end{array}$ \\
\hline $\mathrm{HC} 4 \mathrm{~b}$ & $\begin{array}{l}\text { Propranolol } \\
\text { Atenolol } \\
\text { Hydralazine } \\
\text { Digoxin } \\
\text { Captopril } \\
\text { Methyldopa } \\
\text { Bendroflumethiazide }\end{array}$ & Glibenclamide & Amitriptyline \\
\hline $\begin{array}{l}\text { PNFP } \\
\text { hosp** }\end{array}$ & $\begin{array}{l}\text { Nifedipine } \\
\text { Propranolol } \\
\text { Furosemide } \\
\text { Captopril } \\
\text { Methyldopa }\end{array}$ & $\begin{array}{l}\text { Metformin } \\
\text { Glibenclamide } \\
\text { Insulin Isophane } \\
\text { Insulin Soluble } \\
\text { Insulin Mixtard }\end{array}$ & $\begin{array}{l}\text { Amitriptyline } \\
\text { Imipramine }\end{array}$ \\
\hline
\end{tabular}

*intended for use at HC4 or hospital level, but given to the HC3 by the health sub/district because it was underused and about to expire,

** The PNFP hospital obtained its supplies from Joint Medical Stores.

The one medicine available at all facilities visited was Amuru stated that there was an unused surplus of anpropranolol for hypertension. Otherwise there was tihypertensives and amitriptyline in 2011 that had to be wide variation in medicines for hypertension. Amitrip- destroyed. One HC3 was given a supply of glibenclatyline was the second most common drug, found at mide by the health sub/district because a nearby HC4 four of the six facilities. Three of the six units had no had a surplus that was about to expire.

oral medicine or insulin for diabetes, and two had no

medicine for depression and PTSD (although amitrip- Reviews of the patient registers and interviews with tyline is supplied in the kits for HC3s).

Interviews with staff revealed problems with drug killer. The in-charge of HC3c (where the drug was out stockouts and irregular supplies. The PNFP hospital of stock) explained: "The pattern has changed in the had the widest range of antidiabetic drugs, but reported last three years. Now we give it for constant headache frequent stockouts of metformin and insulin mixtard. and other somatic complaints. We ask, "Does the illness It sometimes ran out of amitriptyline. HC3b and HC3c, interfere with your sleep, do you have bad dreams?" If which reported that amitriptyline was out of stock, re- they say yes, we may give amitriptyline.' Others explained ferred patients to buy it from drug shops. Fluoxetin, a that headache may come from anxiety or depression, selective serotonin reuptake inhibitor drug recommend- and therefore they treated it with amitriptyline.

ed for treatment of PTSD, was not supplied by NMS.

Despite these problems of stockouts, some drugs were According to health workers, many patients sought underused and expired. The district health officer for treatment for hypertension only intermittently. The
Acting in-charge of HC3b said that they gave anti- hypertensive medicines for two weeks, but patients usually did not come back for monitoring and refills at the end of the period.

Medicines for all of these conditions were found at private drug shops and clinics although availability was highly uneven. Near the rural hospital, one drug shop carried anti-hypertensives (propranolol, nifidipine, frusemide) and two private clinics run by a clinical officer and an enrolled nurse respectively managed hypertension with $\mathrm{BP}$ machines and a range of medicines. One also treated diabetes and depression.

Near HC4a were drug shops of which some had BP machines and sold anti-hypertensives. Amitriptyline was found in some drug shops, but diabetes drugs were very rare except at pharmacies and clinics in Gulu town, where those visited stocked glibenclamide and metformin.

\section{Specialized staff and clinics}

Every facility had at least one staff member with formal training in psychiatry. At the HC3 level, there were psychiatric nurses, while the HC4 units also had psychiatric clinical officers. The PNFP hospital, however, had only one psychiatric nurse and no psychiatric clinical officer. Because of understaffing, the staff specialized in psychiatry takes on general duties. At HC4a, which receives outreach support for mental health from $\mathrm{Al}$ derman clinic, we found the outreach staff manning the OPD on one occasion.

Only the two HC4s had dedicated mental health clinic days. The great majority of patients came for treatment of epilepsy. While the one received extra support, the other did not. Interviews with patients showed that those registered at special clinics were given appointment days to come again.

\section{Donor support and research}

Support for the chronic conditions upon which we focused was minimal compared to that for HIV. (The Gulu HMIS report for 2010/11 lists 13 operating partners working in all sub-counties, of which 7 were involved in HIV.) Mental health, including depression and PTSD, attracted most donor attention. In a mapping exercise carried out in the first two months of 2012, we identified $25 \mathrm{NGOs}$ and international organization working in the Acholi region that dealt directly with mental trauma after the war. Some, such as VIVO, do not coordinate with the government health system and we found no evidence of their activities at the health facilities visited. Others such as Transcultural Psycho-Social Organization (TPO) and Alderman Mental Health Clinic have outreach programmes at selected government health centres (TPO reaches HC2 units). The Gulu Diabetes And Hypertension Association, an indigenous NGO, is making efforts to provide training of health workers and patient support, but there are very few resources targeted at cardiovascular conditions and diabetes.

Prevalence studies of PTSD and depression are currently being carried out in northern Uganda using standard questionnaires. Study participants diagnosed with PTSD during the screening are ideally referred to nearby HCs, although this practice was not (yet) evident in the patient reoisters. A research project at $\mathrm{HC} 3 \mathrm{c}$ that was ongoing in January 2012 offered screening for hypertension and diabetes to OPD patients. Over 500 patients were checked in that month alone, and over 100 were referred for treatment. The researcher supplied nifedipine to the unit so that those with hypertension could be given a starting dose. Those diagnosed with diabetes were referred to Gulu Regional Referral Hospital.

\section{Discussion}

While there are no national prevalence figures for hy pertension and diabetes, population-based surveys in several districts suggest that rates are considerably higher than their visibility in public health facilities. For hypertension, a study of people aged 20 and older in Rukungiri district in 2006 found a crude prevalence rate of $30.4 \%{ }^{10}$ A survey in southwestern Uganda in 2009 found a prevalence of $22 \%$. It showed that the vast majority ( $96 \%$ of men and $86 \%$ of women) was not aware of having hypertension. Of those who did know their diagnosis, only about half had controlled blood pressure, suggesting that they were not getting adequate care from health services. ${ }^{11}$ For diabetes, a survey in the same population found prevalences of probable diabetes and probable hyperglycaemia were $0.4 \%$ and $2.8 \%$. Almost $3 / 4$ of those with probable diabetes were unaware that they had the condition. ${ }^{12}$

Prevalence rates for depression are also likely to be much higher than their representation among outpatients at 
public health facilities. A comparison of populations in in availability of diagnostic equipment and medicines. two districts, Adjumani and Bugiri, using the Beck De- Findings from this study show that while all units had pression Inventory, found that $17.4 \%$ were probably functioning BP machines, they were not enough for the clinically depressed. In Adjumani district of northern different services offered, nor were they regularly used. Uganda, which has suffered decades of armed conflict, Glucometers and strips were found in the hospital and the prevalence was $26.3 \%$. Some of the highest rates in one HC4, but that HC4 had no medicine for glucose of depression and PTSD in displaced populations any- control. One HC3 had glibenclamide for diabetes, but where were found among displaced people in Gulu and no glucometer. All units had staff trained to identify Amuru districts in 2006. Using the Hopkins Symptom and treat mental health problems, but two were stocked Checklist-25 and the Harvard Trauma Questionnaire, out of amitriptyline, the principal medication used for $67 \%$ met symptom criteria for depression and 54\% for depression and PTSD.

PTSD. ${ }^{13}$ More recent data on these conditions, in particular as people have been returning to their homes, are still missing.

These population-based research results are in sharp contrast to one key finding of our study: the low visibility of these diagnoses in district HMIS data and in the patient registers at the facilities visited. The prevalence of hypertension at Gulu district OPDs was less than $1 \%$, compared to rates of $22 \%$ and $30 \%$ in the general population elsewhere. The very high rates of depression and PTSD identified 5 years earlier in the districts we studied contrast with the rarity of these diagnoses in the patient registers we examined.

Why are these four conditions so little visible in the outpatient population? One reason could be that people do not recognize them as problems needing biomedical treatment. These diseases do not have conspicuous symptoms like fever or vomiting or herpes rash, which immediately signal sickness. Depression is a much

'quieter', less dramatic disease than psychosis or epilepsy, while PTSD has features that fit with local conceptions of spirit at are to become patients, they must be diagnosed and retained in long-term treatment. This requires equipment and guidelines for making a diagnosis and monitoring treatment, a reliable supply of medicines, health workers with training in chronic care, and procedures fo encouraging the retention of chronic patients. A major finding of this study was that this combination of factors was not in place at the facilities visited with the possible exception of the PNFP hospital.

The Uganda National Treatment Guidelines align management of conditions to levels of care. HC4 and hospitals should be able to diagnose and treat all four of these chronic conditions, while the HC3 is not expected to manage diabetes. This should be reflected

The pattern of diagnoses, both over the years in the district annual reports, and among the 6 health facilities, was uneven, with 'clumping' of diagnoses in certain years and at certain units. This unevenness might be due to several factors. Staff members working in OPD at a particular time may be more alert to particular symptoms and diagnostic categories, because of special training or recent workshops. Diagnostic equipment may be available at some times and not others. Externally funded projects, such as the outreach programmes for mental health at HC4a, may be active in given periods. Since such projects usually have a limited duration, projects would also have the same effect in drawing attention to a disease for a short intensive interval.

\section{Conclusion}

Although international and national policy emphasiz es the growing need for care of hypertension, diabetes, and depression, these conditions are not very visible within the public health services in northern Uganda. This is so despite research that suggests they are highly prevalent in the population. It is striking that PTSD, which has attracted the attention of researchers and NGOs as a consequence of the protracted conflict and encampment, is hardly visible as a diagnosis in the patient registers.

One of the lessons of the response to HIV was that dedicated clinic days for monitoring and medicine refills support adherence. HC4s and most hospitals have special clinics for mental health, which give patients appointments for follow-up. It would be possible to establish similar clinics for hypertension and diabetes at the same level of service. This would contribute to regularizing care and medication, and would make these two NCDs more visible to both health workers and patients. accessible services provided by trained staff. their effects may appear to be intermittent. Research
Another lesson is the necessity of constantly available diagnostic instruments and awareness that screening must be a ticket to continuing treatment for those found to have the disease. Periodic screening drives for hypertension and diabetes, such as those organized fo special events (e.g Diabetes Day), help to raise awareness and to identify people who had been unaware of their conditions. But screening alone is not enough; follow-up must be ensured in the form of available and

6. WHO/MOH 2006 WHO-AIMS report on the mental health system in Uganda. WHO, MoH Uganda; see also: Ssebunnya J, Kigozi F, Ndyanabangi S. Developing a national mental health policy: a case study from Uganda. PLoS Med 2012; 9(10):e1001319.

. Daar A S., et al. Grand Challenges in Chronic Non-Communicable Diseases: The Top 20 Policy and Research Priorities for Conditions Such as Diabetes, Stroke and Heart Disease. Nature 2007; 450:494-496 8. WHO. Cluster Strategy for Noncommunicable Diseases and Mental Health 2008-2013. WHO/ $\mathrm{NMH} / 2009$.2. Geneva: WHO.

\section{Acknowledgements}

The study took place under a larger collaborative research training programme, 'Changing Human Security: Recovery From Armed Conflict In Northern Uganda' for which clearance was granted by UNCST. We gratefully acknowledge funding from Danida, and the guidance of Professor Emilio Ovuga and Dr. Thomas Oyok

\section{References}

1. Boutayeb A. The double burden of communicable and noncommunicable diseases in developing countries. Transactions of the Royal Society of Tropical Medicine and Hygiene 2006; 100: 191-109.

2. Alberti G. Noncommunicable diseases: tomorrow's pandemics. Bulletin of the World Health Organization 2001; 79 (10): 907

3. WHO, "First NCDnet Global Forum," 2010, http:// www.who.int/nmh/newsletter_apr2010.pdf.

4. $\mathrm{MoH}$ and Republic of Uganda. Health Sector Strategic Plan II: 2005/06-2009/2010, Vol. I. Kampala: Republic of Uganda, 2005.

5. WHO The global burden of disease: 2004 update. World Health Organization, 2008; p. 50-51.
9. Maher D, Sekajugo J, Harries AD, Grosskurth H. Research needs for an improved primary care response to chronic non-communicable diseases in Africa. Tropical Medicine \& International Health 2010;15 (2):176-181.

10. Wamala JF, Karyabakabo Z, Ndunguse D, Guwatudde D. Prevalence factors associated with hypertension in Rukungiri District, Uganda - a community-based study. African Health Sciences 2009; 9 (3):153-160.

11. Maher D, Waswaa L, Baisley K, Karabarinde A, Un win, N. Epidemiology of hypertension in low-income countries: a cross-sectional population-based survey in rural Uganda. Journal of Hypertension 2011; 29:10611068

12. Maher D, Waswaa L, Baisley K, Karabarinde A, Unwin, N, Grosskurth H.. Distribution of hyperglycaemia and related cardiovascular disease risk factors in low-income countries: a cross-sectional population-based survey in rural Uganda. International Journal of Epidemiology 2011; 40:160-171.

13. Roberts B, Kaducu FO, Browne J, Oyok T, Sondorp E.. Factors associated with post- traumatic stress disorder and depression amongst internally displaced persons in northern Uganda. BMC Psychiatry 2008; 8 (38). 